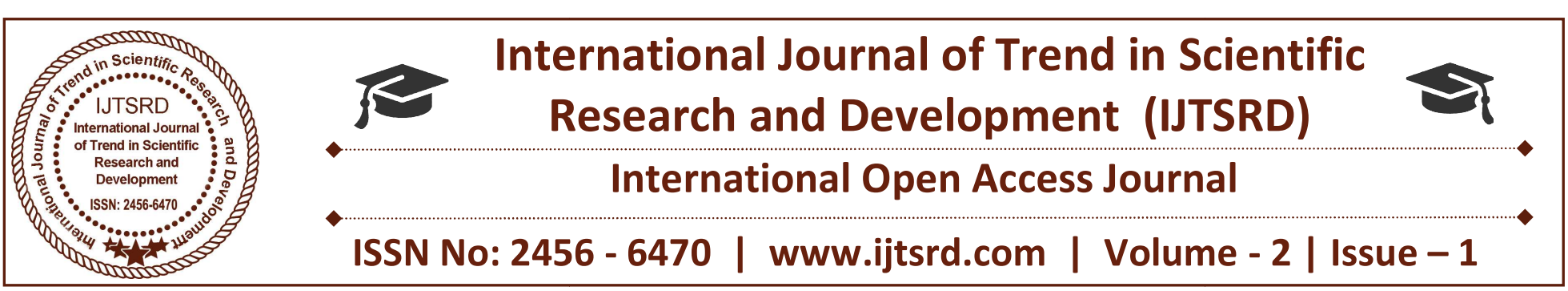

\title{
Occupancy-based Surveillance with Wireless Camera Network for Public Safety: Creating Safe Places with Internet of Things to Live in Urban
}

\author{
Hakkı Soy \\ Electrical and Electronics Engineering Department \\ Necmettin Erbakan University \\ Konya, Turkey
}

\author{
Yüksel Çelik \\ Computer Engineering Department \\ Karabük University \\ Karabük, Turkey
}

\begin{abstract}
The monitoring of public areas with video cameras is becoming increasingly important due to the security concern around the world. Real-time surveillance of people and their activities in urban environments is becoming increasingly important in the context of public safety. The advance of wireless technologies has expanded the scope and capabilities of camera networks, which now can be used as intelligent platforms to provide public safety. This paper aims to present an occupancy-based surveillance system at the intersection of Internet of Things and wireless camera networks.
\end{abstract}

Keyword: public safety; camera network; wireless communication; occupancy detection

\section{INTRODUCTION}

Cities make up only two percent of the earth's surface, yet they are home to over half of the world's population [1]. People generally choose to live in cities to improve their life quality. So, the urban places are important for public safety [2]. The public safety is defined as any entity or services that protect the life and values of citizens against dangers from the side of violent operations of people and forces of nature. It aims to keep citizens and public areas safe with faster response, improved operational efficiency and reduced costs [3]. In parallel with the continuous increase of urbanization and crime rates, the security and justice institutions need advanced technologies to improve the safety of the urban places [4].
Police and local law enforcement authorities collect real-time information about the people, objects and environment by combining data from various sensors with different modalities. But, image sensor is a most important sensing capability to be used for surveillance of public areas [5]. Video surveillance systems consist of a number of spatially distributed cameras that continuously monitor the observed area. The surveillance of public areas is intended to deter the crime and aid in protecting the safety and security. By this way, the criminal activities can be monitored and recorded in the event of an emergency situation [6].

In surveillance applications, timely detection of abnormal events has a critical importance [7]. But, securing large areas with adequate camera coverage can be challenging task that requires a systematic approach. The camera networks are the fastest growing application of video surveillance, where lots of cameras monitor large scale areas. Today, the camera networks are deployed in many sites that require surveillance and security, such as airports, railway stations, hospitals, nuclear power plants, campus, military bases and border control $[8,9]$.

Camera specifications play an important role in efficiency of surveillance applications. The image sensor type is an important factor that affect the performance of camera [10]. CMOS (complementary metal oxide semiconductor) and CCD (charge coupled device) image sensors are two different technologies for capturing images digitally [11]. Image sensors produce large data per sample based on image size. It is important to note that larger amounts of data require 
more processing and communications, increase analysis complexity and exhaust more resources. The wireless communication technology removes the need for wires to transmit video signal to the remote-control room. Besides, image processing capability of digital cameras dramatically reduces the communication bandwidth demands by detecting only abnormal or suspicious behaviors [12].

Fully digital IP-based cameras provide an improved surveillance experience with their pan, tilt and zoom (PTZ) functionalities. Since surveillance systems have to cover very large areas, the video cameras directed towards different viewing zones where there could be both overlapping and non-overlapping fields of view of the cameras. When the cameras have non-overlapping field of views, there exist unobserved areas in the camera network. PTZ cameras can pan left and right, tilt up and down and zoom in or out through manual or automatic control. They can be used to cover much larger areas than stationary cameras $[8,13]$.

In recent years, as wireless and micro-electronic technologies evolve, the surveillance systems have shift towards embedded platforms which have more adaptability than traditional PC-based solutions. With the latest advances, it is becoming more feasible to develop the wireless camera networks (WCN) that consist of low-cost, low-power camera nodes (CNs) with limited resources [12]. Moreover, video cameras can be integrated to the Internet of Things (IoT) platforms to make the surveillance applications more attractive. In the near future, IoT will enable a variety of new system designs that were previously impossible in video surveillance applications up to now [14].

In this study, we present the research conducted on surveillance system with WCN. In our system, the cameras are triggered only when occupancy is detected by sensors. The proposed system uses the ZigBee and IoT technologies to produce an automated surveillance. The rest of study is organized as follows. Section II gives the framework of proposed system. Section III explain hardware design details. Finally, we conclude the study in section IV.

\section{System Overview}

The proposed system consists of several camera nodes (CNs) and certain number of occupancy nodes (ONs) besides the sink. Each $\mathrm{CN}$ comprises image sensor, microcontroller, ZigBee RF transceiver and Wi-Fi module. The CNs capture, process and transmit visual data (images and/or videos) to common sink for analysis. The $\mathrm{CNs}$ are also combined with servo motors to steer the onboard pan-tilt mechanism. The ONs include motion sensor and ZigBee RF transceiver to automate the tracking function via rotating pan-tilt mechanism of CNs. The ONs are powered with batteries, while the CNs can be operated with batteries or AC adapter. The energy-unconstrained sink node is equipped with ZigBee RF transceiver to receive or send the data.

At this point, we propose two different network architectures to gather visual data from the CNs. In "automated" network architecture, the ONs detect the presence of people and report it to neighbor $\mathrm{CNs}$ through ZigBee connection. The CNs estimate the occupancy areas and turn cameras to the appropriate position where motion signals were taken. After that, the visual data transferred from $\mathrm{CNs}$ to the cloud server via IP connection. In this setup, PTZ direction estimation algorithm is run on the CNs as shown in Fig. 1. On the hand, the sink coordinates the overall surveillance in "semi-automated" network architecture. The ONs detect the presence of people and report it to sink. The sink runs the PTZ direction estimation algorithm and send position data to direct the related cameras to the occupancy areas. The communication between the ONs, sink and CNs is established through ZigBee connection. The CNs send the visual data to the cloud server via IP connection as shown in Fig. 2.

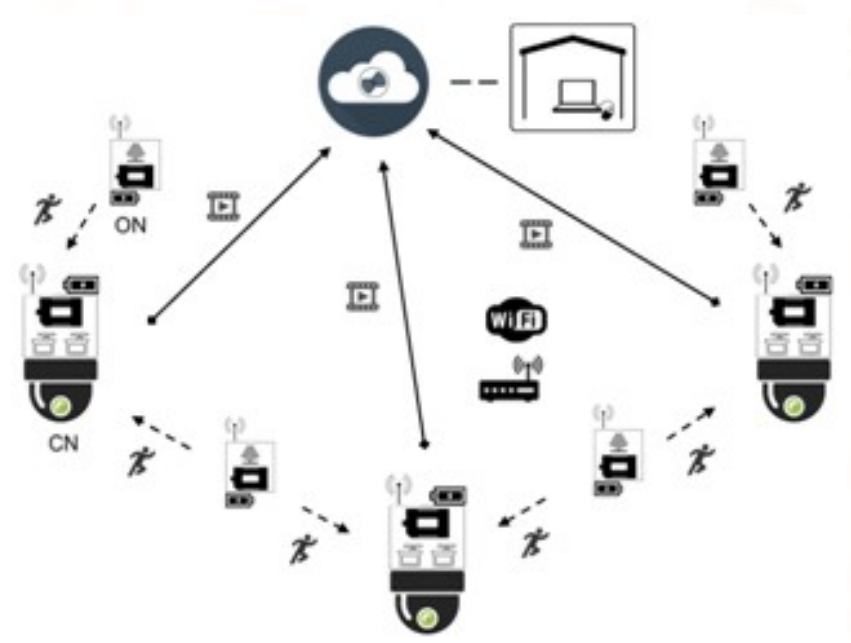

Fig. 1. Automated network architecture. 


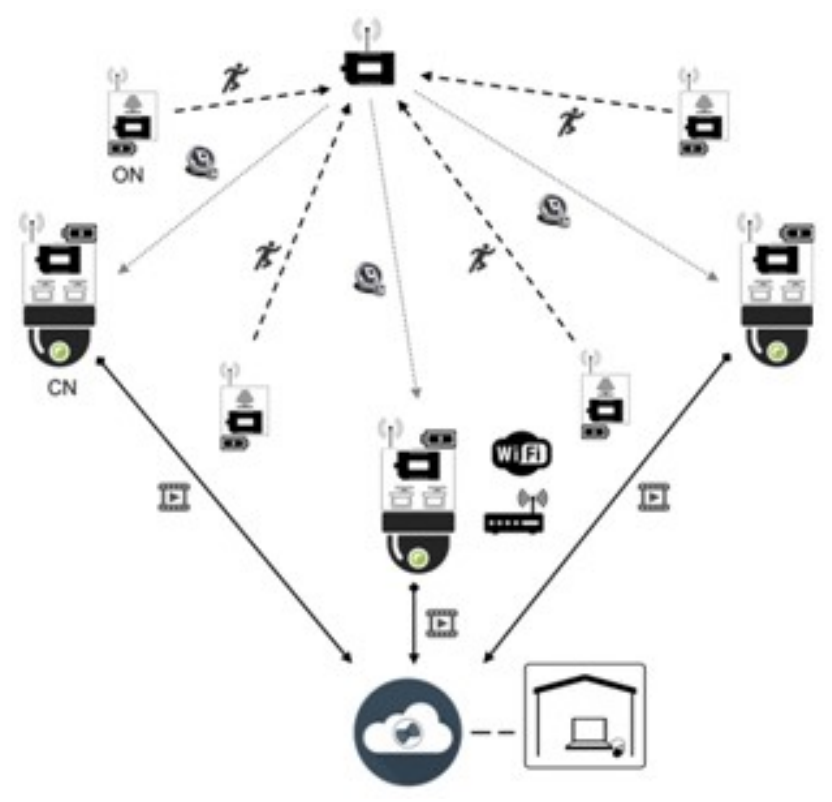

Fig. 2. Semi-automated network architecture.

Depending on the application requirements and working environment, one of these architectures may be used for surveillance. It can be considered that the latency is minimized in automated architecture due to the occupancy signal is conveyed directly from ONs to CNs. Note also that the automated architecture works independently of the sink. But despite that, the CNs have higher load (direction estimation, PTZ calculation and video streaming). Clearly, the sink can lighten the load on the CNs in semi-automated architecture.

The aim of our system is to continuously monitor the sensitive zones around the public sites. We assume that the ONs, sink and CNs are deployed in the coverage area regularly. When the ONs detect suspicious activity, broadcast the alarm mesage with their address to the CNs either directly or indirectly via sink node. In automated architecture, some CNs receive the alarm and estimate the event location from sender $\mathrm{ON}$ address. It is assumed that each $\mathrm{CN}$ knows the exact location of all the ONs. So, the CNs direct the PTZ cameras to the occupancy area by actuated their own servo motors. Whereas, in semi-automated architecture, the alarm messages are received by sink and the CNs only wait pan-tilt orientation commands from sink. In both cases, the CNs are positioned to focus on target and record the action. Fig. 3 shows the application scenario of proposed occupancy based surveillance system for public safety.
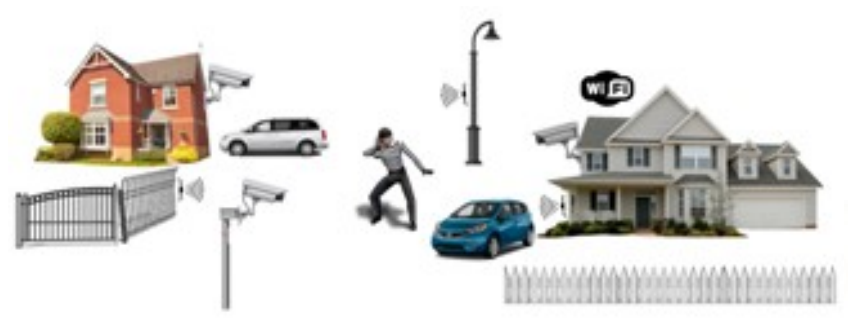

Fig. 3. Occupancy-based surveillance with WCN.

\section{Hardaware Design}

The CN hardware design is developed on Orange Pi PC Plus single board computer which is designed to provide advanced functions with low-cost computer hardware. As shown in Fig. 4, Orange Pi PC Plus board has on-board Wi-Fi module to easily add a wireless connection to the Internet and provides a perfect base platform for creating Internet-connected things. It uses the AllWinner H3 system on chip (SoC) platform and has 1GB DDR3 SDRAM. Besides, 8-bit YUV422 CMOS sensor interface is supported by Orange Pi PC Plus and the camera serial interface (CSI) port is added to connect the camera.

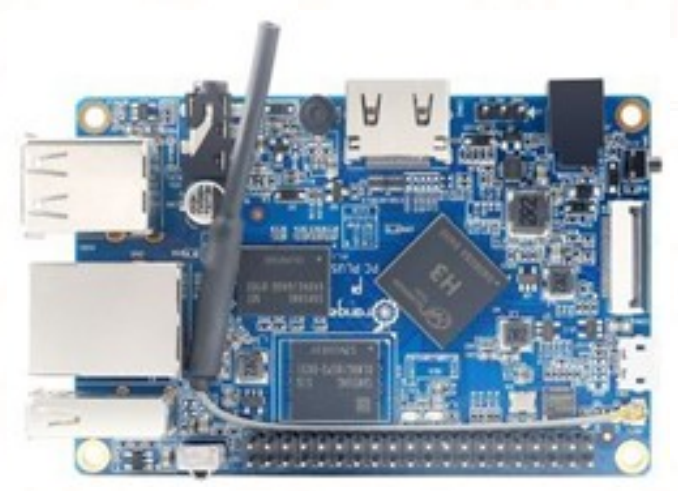

Fig. 4. Orange Pi PC Plus board.

In $\mathrm{CN}$ hardware design, we use the Orange $\mathrm{Pi}$ compatible $2 \mathrm{MP}$ color camera with wide-angle image sensor that can be used in surveillance applications. The camera placed over the pan-tilt mechanism and the pan-tilt mechanism is directly actuated by the servomotors which is mounted to the CN. Fig. 5 shows the Orange Pi camera and pan-tilt mechanism with servo motors to track targets on the surveillance area.

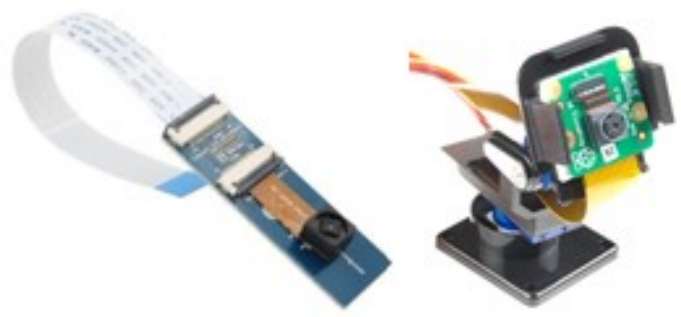

Fig. 5. Camera and pan-tilt mechanism. 
The ON and sink designs are built on the low-cost Arduino Nano board which has an Atmel ATmega328 microcontroller. We use low-cost Pdlux Electronic PDV1 microwave motion sensor for occupancy detection. PD-V1 sensor operates as C-Band Bi-Static Doppler radar in $5.8 \mathrm{GHz}$ frequency band. It has a built-in coaxial resonator oscillator $(\mathrm{CRO})$ and covers an area of $360^{\circ}$ for occupancy detection applications. This module uses the built-in amplifier circuit to make signal processing simpler. The motion signal is send to the microcontroller through one data wire. Fig. 6 shows the $\mathrm{PD}-\mathrm{V} 1$ sensor and $360^{\circ}$ antenna radiation pattern.

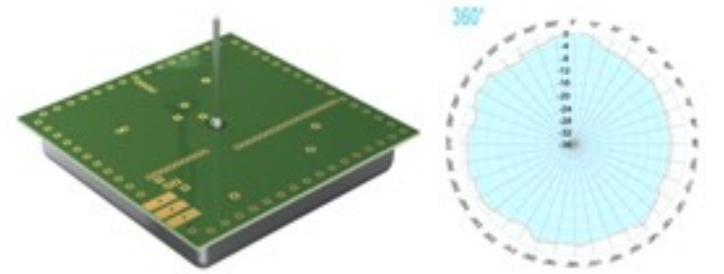

Fig. 6. Pdlux PD-V1 motion sensor and radiation pattern.

We use the Nordic Semiconductor nRF24L01+ (with power amplifier and SMA antenna) low-power transceiver to establish wireless communication links between the ONs, sink and CNs. The nRF24L01 transceiver operates in the unlicensed $2.4 \mathrm{GHz}$ ISM band and communicate with different $(250 \mathrm{Kbps}, 1$ or 2 Mbps) baud rates. The transmission distance is close to about 1000 meters (for $250 \mathrm{Kbps}$ baud rate) in line-ofsight case. It has an antenna integrated on the PCB, quartz oscillator and SPI interface to microcontroller connection. Fig. 7 shows the testbed setup of the CN. Fig. 8 shows the testbed setups of the ON and sink. In these designs, Arduino Nano IO Shield with nRF24L01 socket is used to integrate the components.

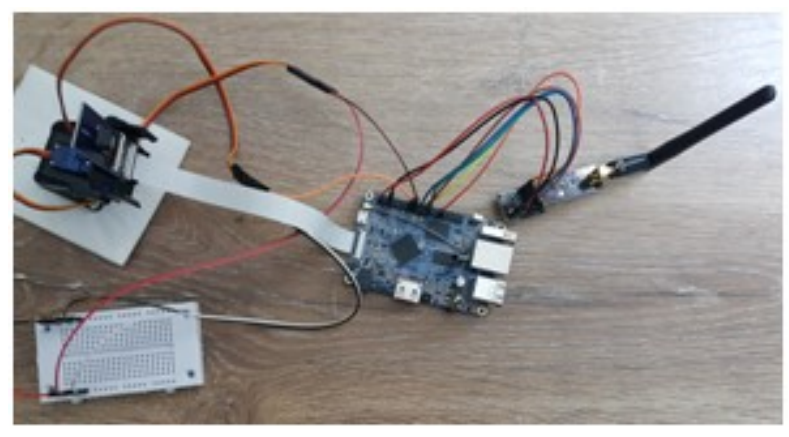

Fig. 7. Camera node testbed setup.

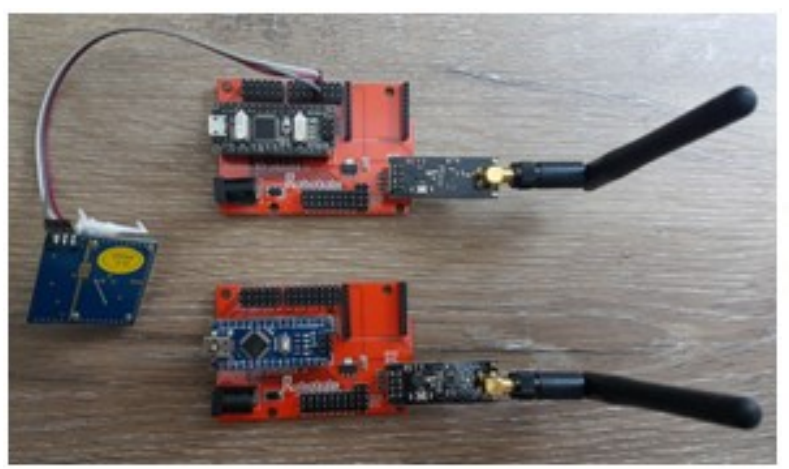

Fig. 8. Occupancy node and sink testbed setups.

The video streams of CNs accessible from anywhere in the world by using required software, such as MJPEG streamer and Ngrok [15]. MJPG-streamer is the simple video streamer for video monitoring and surveillance applications [16]. Ngrok is the software service that allowing users to access the local streaming server and makes it available on the web [17]. Fig. 9 shows the flowchart of the operation of $\mathrm{ONs}$ and $\mathrm{CNs}$ in automated system architecture.
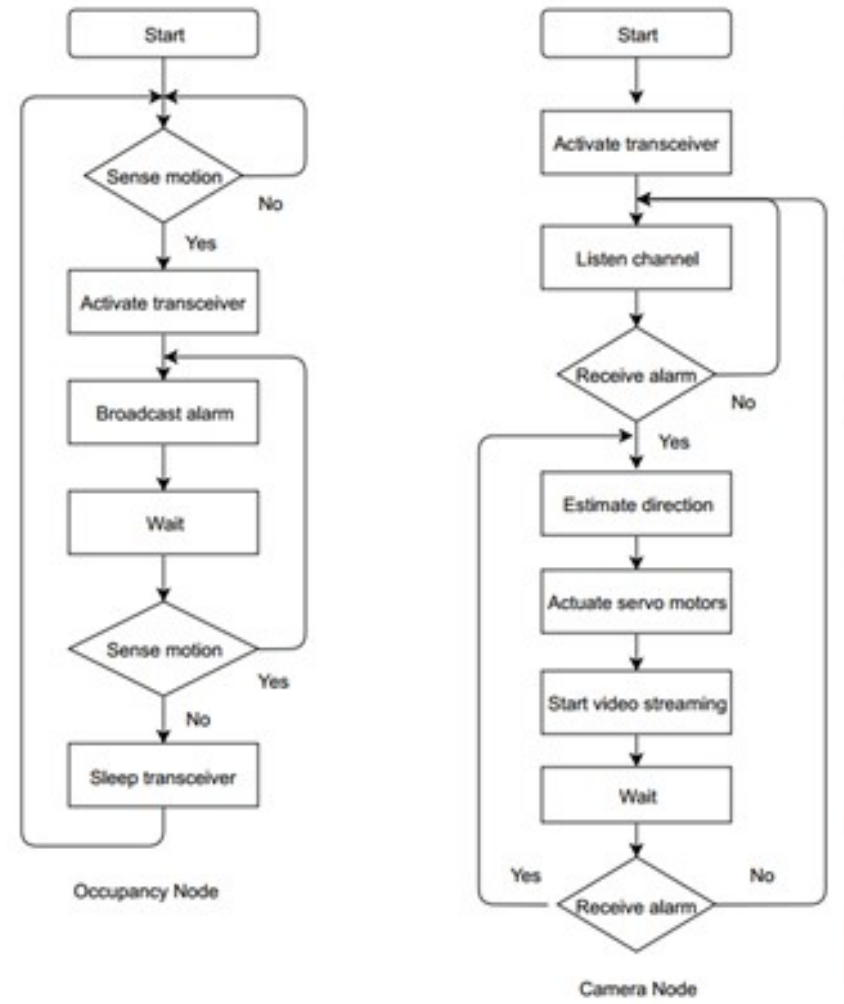

Fig. 9. Flowchart of the ONs and CNs in automated mode.

\section{Conclusion}

Over the past few years, IoT grows rapidly and offers enormous opportunities for public safety services. In addition to traditional network enabled devices, video cameras can be integrated to the IoT platforms to capture emergency events instantly. This study presents the design of the wireless network of video cameras 
and motion sensors. We propose two different network architectures to perform automated surveillance by using pan-tilt functions of video cameras. In proposed system, the video streaming is started only when the alarm is triggered by the motion sensors. Servo motors orientate the cameras to the target area. In our future works, we will extend the proposed system with direction estimation algorithms.

\section{References}

1) European Union, Making our cities attractive and sustainable. Publications Office of the European Union, 2010.

2) S. Roche and A. Rajabifard, "Sensing places' life to make city smarter," in Proceedings of ACM SIGKDD International Workshop on Urban Computing (UrbComp 2012), Beijing, China, August 2012.

3) D. Camara and N. Nikaein, Wireless public safety networks, Volume 1, Overview and challenges. ISTE Press - Elsevier, 2015.

4) United Nations, Enhancing urban safety and security. UN-Habitat, 2007.

5) V. Zeljkovic, Video Surveillance Techniques and Technologies. IGI Publishing 2013.

6) Urban Institute and Community Oriented Policing Services, Using Public Surveillance Systems for Crime Control and Prevention: A Practical Guide for Law Enforcement and Their Municipal Partners. Urban Institute, 2011.
7) S. Gong, C.C. Loy, T. Xiang, "Security and Surveillance," in: Visual Analysis of Humans. Editors: T. Moeslund, A. Hilton, V. Krüger, L. Sigal, Springer, 2011.

8) A. Del Bimbo, F. Dini, A. Grifoni, F. Pernici, "PanTilt-Zoom Camera Networks", in Multi-Camera Networks: Principles and Applications. Editors: H. Aghajan and A. Cavallaro, Academic Press, 2009.

9) A. K. Roy-Chowdhury and B. Song, Camera Networks: The Acquisition and Analysis of Videos over Wide Areas. Morgan \& Claypool, 2012.

10) A. C. Caputo, Digital Video Surveillance and Security. Butterworth-Heinemann, 2014.

11) Axis Communications, "CCD and CMOS sensor technology (Technical white paper)," 2010.

12) M. Al Najjar, M. Ghantous, and M. Bayoumi, Video Surveillance for Sensor Platforms. Springer, 2014.

13) K.-Y. Li et al., "A web-based, real-time video surveillance system by leveraging PTZ cameras," IEEE Tenth International Conference on Intelligent Sensors, Sensor Networks and Information Processing (ISSNIP), Singapore, 2015.

14) V. Haribaabu and S. J. James, "Intelligent Surveillance System using Internet of Things," International Journal of Computer Application, vol. 2, pp. 25-29, 2013.

15) M. Schwartz, Building a Wireless Security Camera with Arduino. Open Home Automation, 2016.

16) https://github.com/jacksonliam/mjpg-streamer

17) https://ngrok.com/ 